\title{
Correction to: Countering Biases in Risk Analysis
}

\section{Correction to:}

Chapter 2 in: S. Hunziker, Enterprise Risk Management, https://doi.org/10.1007/978-3-658-33523-6_2

Despite careful preparation of our books, errors cannot be avoided, so we would like to point out the following: The numbering of the headings and the table of contents has been corrected. 